\title{
EL TEXTO ETNOGRÁFICO Y LA PROBLEMÁTICA INDÍGENA. LAS POSIBILIDADES DE UNA ESCRITURA DIFERENCIADA EN LA INVESTIGACIÓN ANTROPOLÓGICA DE ANNE CHAPMAN
}

\author{
THE ETHNOGRAPHIC TEXT AND INDIGENOUS ISSUES. \\ THE POSSIBILITIES OF A HANDWRITING DIFFERENTIATED \\ IN THE ANTHROPOLOGICAL RESEARCH BY ANNE CHAPMAN
}

\section{GABRIELA ÁLVAREZ GAMBOA ${ }^{1}$}

\section{RESUMEN}

El texto etnográfico fue preponderante a fines del siglo XIX, donde el vencido es (re) posicionado como objeto de estudio, para dar muestra al mundo de una realidad que se fragmentaba a pedazos. Son discursos que se manifiestan mediante recursos formales que constituyen las convenciones del género. Según lo anterior, el objetivo de este trabajo es revisar el discurso de la antropóloga Anne Chapman, en relación a la etnografía tradicional, y la incorporación de otras mecánicas textuales que permitan entrever una escritura diferenciada.

Palabras clave: Discurso, etnografía, Patagonia.

\section{ABSTRACT}

The ethnographic text was predominant in the late nineteenth century, where the defeated were re-positioned as an object of study to show the world a reality which was fragmented into pieces. These are discourses, manifested through formal resources, that constitute the conventions of the genre. According to the above, the objective of this paper is to review the discourse of anthropologist Anne Chapman and compare it to traditional ethnographic discourse as well as to the incorporation of other textual mechanics in order to glimpse a differentiated writing.

Keywords: Speech, ethnography, Patagonia.

Recibido: 04.11.11. Aceptado: 18.10.13.

${ }^{1}$ Candidata a Doctora en Estudios Americanos. Universidad de Santiago de Chile. Santiago, Chile. Becaria Conicyt. E-mail: gabrielaalvarez5121@hotmail.com 
El poder de la mujer es corto, es de aquellos que partieron ese, no es corto.

(Canto chamánico de Lola Kiepja)

E L TeXto etNOgRáfico fue preponderante a fines del siglo XIX en la Patagonia, espacio en el cual el vencido es (re)posicionado como objeto de estudio para mostrar al mundo una realidad que se fragmentaba en pedazos. Con anterioridad, la recopilación de datos sobre la Patagonia cumplía la función de remover el carácter desértico asignado a la geografía (como imaginario), y reunir información en el afán de implantar una economía agro-exportadora. En este contexto, el indígena fue generalmente descrito como un "enemigo" a los propósitos del Estado. Sin embargo, la etnografía estudió su derrota dentro de la narración progresista, en la necesidad de retener el máximo de antecedentes para construir una descripción fidedigna de una cultura ad portas de la extinción.

El discurso etnográfico especifica una cultura de la manera más verosímil posible. Su antecedente más cercano son los relatos de viajes, ya que la experiencia en terreno fue su metodología más destacable. George Marcus y Michael J. Fischer (1986) estudiaron los elementos centrales de la tradición etnográfica, además de reflexionar sobre las posibilidades de la representación en estos tiempos, y de cómo es posible confeccionar un texto más acorde con las discusiones actuales. Análisis que destaca los ejercicios de poder en la construcción discursiva, dado que la narración sobre el otro se sustenta en patrones ideológicos según la cultura del sujeto productor. Precisamente, Sergio Poblete (1999) cuestiona la objetividad como característica autoimpuesta del texto etnográfico porque éste sería una representación ficcional de las experiencias del autor, es decir, una: "composición lingüística de elementos de la experiencia del descriptor que tiene como objetivo evocar en el lector experiencias semejantes a las del autor. La ficción etnográfica genera, en base a ciertas convenciones y recursos lingüísticos, evocaciones con sentido que un sujeto puede considerar representaciones de la realidad" (Poblete, 1999: 212).

Los siguientes elementos despliegan los recursos que constituyen las convenciones del género. Primero, registrar un desarrollo general del objeto social a través de la división en unidades de análisis (origen, organización socioeconómica, geografía, etc.). Segundo, la ausencia del sujeto etnógrafo mediante el uso de un relato impersonal o, bien, un narrador omnisciente para afirmar la autoridad del investigador. Tercero, enfatizar el trabajo en terreno con la delimitación de las fuentes o los marcadores simbólicos (fotografía, entrevistas, cuadros, etc.). Finalmente, recrear situaciones cotidia- 
nas que subrayen la inclusión "natural" del observador en el campo social analizado. De acuerdo a lo anterior, el objetivo del presente trabajo será revisar otras mecánicas textuales en Los selk’nam. La vida de los onas (1986), de Anne Chapman, que permita entrever una escritura diferenciada.

En los años 60, Anne Chapman fue parte del equipo de investigación de la arqueóloga Annete Laming-Emperaire en la Patagonia, contexto en el cual conoció a Lola Kiepja -considerada la última chamán selk'nam-, con quien le unió, en palabras de la misma autora, una profunda amistad. Gracias a Lola, Chapman estuvo al tanto de la existencia de otros miembros de la comunidad como Ángela Loij. Ambas fueron los pilares fundamentales en el estudio llevado a cabo por la investigadora en torno a la Patagonia y sus habitantes. Cierran este cuadro de informantes, los mestizos selk'nam y haush: Federico Echeuline, Esteban Ishtón y Luis Garibaldi.

La investigación de Chapman tenía como objetivo, al igual que los textos clásicos etnográficos, dar cuenta de los usos culturales de la sociedad selk'nam, operación que demostró las diferencias y dificultades con sus antecesores. Describir implicaba "estar allí", tomar nota de los aspectos más representativos y situarlos en un ordenamiento formal mediante los recursos apuntados anteriormente. Chapman, que realizó un trabajo en terreno de más de diez años, no fue una actriz presencial de las prácticas culturales del otro. El hain, tópico central en sus reflexiones, había desaparecido como ejercicio cultural. Por lo tanto, nos enfrentamos a una maniobra representacional cuya base es el vacío o, más bien, el fragmento que la escritura intentaba narrar a través de la recreación de la memoria de cada uno de sus sobrevivientes. Los indígenas tuvieron que traducir a la investigadora tradiciones que no practican en su totalidad, porque habían sobrellevado un largo proceso de marginalización, en que los episodios de su cultura de origen muchas veces ocupaban espacios sólo en la remembranza cotidiana. En este sentido, fue una lucha contra el olvido en medio de una linealidad temporal impuesta por el hombre blanco.

La autora intentó releer una oralidad que enmarque las historias de los últimos indígenas selk'nam. Las simbologías y ceremonias decaídas vuelven a ser estudiadas, incorporadas en el discurso científico en una ficción narrativa de la experiencia del otro, en síntesis: “... lo que el etnógrafo aprehende son las formas simbólicas que los 'nativos' usan para conceptuar su realidad, por tanto lo que una descripción representa son las interpretaciones de los nativos a su mundo", es decir, una "interpretación de una interpretación" (Poblete, 1999: 230).

Una de las estrategias de los textos etnográficos era apoyarse en refe-

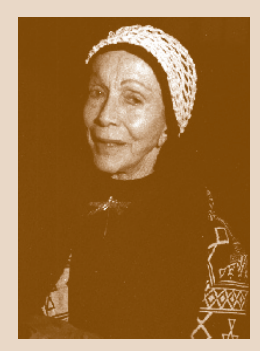

A. Chapman

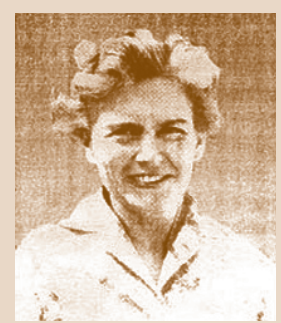

A. Laming 


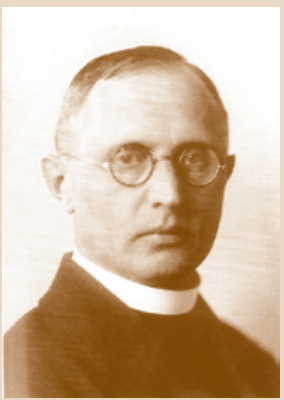

M. Gusinde

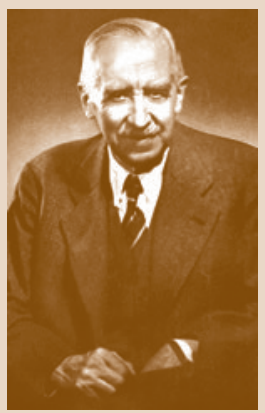

L. E. Bridges rencias anteriores. El intertexto fue una maniobra común para retomar y sugerir algún aporte argumentativo, y/o acudir a la sobreinformación con el objeto de enfatizar lo enunciado. Anne Chapman manejó en su investigación dos fuentes centrales: Martín Gusinde (1982) y Lucas Esteban Bridges (1952), autores que en distintos momentos se interesaron en describir textual y visualmente las distintas comunidades indígenas en la zona patagónica. Ahora bien, la temática central en el texto de Chapman (1986) fue la ceremonia hain, por eso las citas apelan sobre todo a Martín Gusinde, que convivió con los selk'nam en la última escenificación de esta ceremonia en el año 1923.

¿Por qué eligió a Gusinde? Además de lo indicado, el investigador fue parte de un aparataje teórico que pretendió probar la autora en su discurso: que Gusinde describió a la sociedad selk'nam en concordancia con los patrones falocéntricos. Entonces, Chapman buscó constituir un constructo teórico que fisure el discurso dominante en lo que respecta al lugar de la mujer. Por lo tanto, su intención va más allá de recomponer un escenario cultural determinado, fue recontextualizar el papel de la mujer selk'nam en la ceremonia hain (a pesar del "secreto" impuesto a las mujeres).

Chapman se hizo cargo de sus desafíos antropológicos mediante la organización textual, con la inclusión de incógnitas en los encabezados del texto. La operación era la siguiente: discutir la pregunta autoimpuesta y relacionarla inmediatamente con las citas de los autores nombrados y, así, llegar a una conclusión que exponga los intereses de su investigación, (re) contemplar la esfera de la mujer indígena. En el primer capítulo la interrogante era ¿Fue "simple" la sociedad selk'nam? La autora contrapone esta interrogante frente a las nociones rígidas como "banda" y "sociedad patrolineal-flexible", que logró resolver, subrayando ciertas refutaciones teóricas y el contraste con otros criterios de lecturas. Por ejemplo, la exposición de los pares tecnología versus religión, la autora gira la atención hacia la menor complejidad de las sociedades industriales -en el plano religioso-, en comparación con las sociedades selk'nam.

Respecto al ejercicio del poder, el tema fue discutido de acuerdo a los grados de continuidad y discontinuidad en su acceso, así como la igualdad en su obtención, espacios importantes dentro de la problemática femenina presentada en el texto. Chapman realizó un catastro de los rangos jerárquicos y se detuvo en aquellas instancias en que las funciones son similares y diferentes en ambos géneros. La posición social de la mujer es el subtítulo del segundo capítulo, la autora introduce su desacuerdo con la postura de Gusinde sobre la sumisión de la mujer al hombre. Lectura que se sostuvo 
tomando como dato los castigos provocados por el esposo -especialmente durante la ceremonia hain-, y el papel central del hombre en la estructura económica. Chapman afirmó que tal postulado deja en evidencia la proyección del autor: “... Gusinde tenía una visión de las relaciones hombremujer típica de su época y por eso le era difícil concebir la igualdad entre los sexos" (Chapman, 1986: 96).

Y frente a la demostración del sometimiento de la mujer selk'nam en las citas constantes de Bridges, Gusinde y Federico Echeuline, la autora extrapoló una lectura más móvil de la sociedad indígena: aceptó que la mujer selk’nam tenía una posición inferior al hombre; no obstante, esta idea estaba muy lejana a la concepción de esclava o "víctima del sistema":

[...] si bien ella admitía la necesidad que tenían los hombres de dominarla y humillarla durante la celebración del hain, no era por eso mismo víctima del "sistema". Sobre todo, era muy digna; capaz de enfrentar la autoridad masculina, de abandonar a un marido abusador, de desafiar a un chamán varón midiendo sus propios poderes con los de él. Si las circunstancias lo requerían, ella sola podía cazar guanacos con la ayuda de perros o alimentar a su familia con peces, moluscos y plantas. Las mujeres no eran simples reproductoras ni resignadas trabajadoras, ni tampoco esposas sumisas (Chapman, 1986: 99).

En la interrogante ¿La sociedad selk'nam era igualitaria? cuestionó los supuestos antropológicos en relación a dar cuenta cabalmente de una caracterización certera de la sociedad selk'nam según el término "cazadoresrecolectores", demarcación que constituye más bien una idea tipo que describiría a los indígenas en función de que el hombre ocupaba un lugar dominante en la comunidad. Para la autora, los prototipos enunciados por la teoría antropológica no presentaban una correlación total en la colectividades selk'nam, porque sus estructuras fueron más flexibles o temporalmente sus actitudes sociales lograban encasillar en la terminología científica. Por lo mismo, reiteró la visión machista de Gusinde, que puntualizaba como "naturales" los procesos de subordinación, en cuanto a que los roles y los deberes de los sujetos serían identificables según su sexo:

[...] Los prejuicios masculinos pesan en la antropología porque pesan en nuestra sociedad. Los estudios sociales deben, a mi juicio, conceder igual valor a ambos sexos asignando al papel de la mujer la misma importancia que al del hombre. Si se menosprecian las actividades y la condición social de la mujer, o si se los describe y analiza sin el rigor debido, los procesos sociales son falseados (Chapman, 1986: 100).

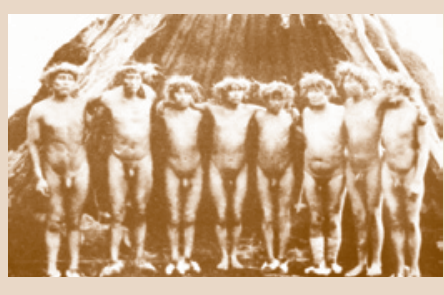

Ceremonia hain 
La exploración de los componentes de la ceremonia hain implicó diferenciaciones que entendiera el conjunto de la sociedad selk'nam en su sentido ritual-religioso, así como la comprensión del papel de cada actor en su conjunto. En primer lugar, la autora indagó en los dos mitos más significativos de los selk'nam titulados como El mito del matriarcado y $\mathrm{El}$ primer hain de los hombres.

En resumen, el hain fue una práctica cultural ejercida por los hombres y las mujeres en temporalidades distintas. El hain femenino (tiempo mítico) fue el momento de iniciación de las mujeres hacia la adultez y el control de la autoridad dentro del grupo. Durante el desarrollo de la ceremonia los espíritus cumplían la función de mantener a los hombres sometidos al poderío de las mujeres, por eso rondaban los hogares asustando, amenazando pero también divirtiéndolos. El secreto consistía en jamás revelar que eran ellas disfrazadas de los espíritus. En cambio el hain masculino (fue una forma de ordenamiento social) ocurrió una vez que descubrieron el engaño de las mujeres. La decisión de los hombres ante tal hallazgo fue radical: matar a todas las mujeres (a excepción de Luna) y, tras un largo periplo cargando su inabarcable pena, resuelven reiniciar el rito nuevamente.

Chapman problematizó la ceremonia con la revisión de los elementos que la constituye, especialmente le interesó el ocultamiento de la identidad de los espíritus a las mujeres, las ambivalencias de sus caracteres y la participación activa de sus miembros en cada instancia del desarrollo del hain. La representación de sus dioses no fue considerado por los selk'nam un engaño, sino apeló a la creencia profunda de que los espíritus ocultaban el pasado mítico de las mujeres:

La ideología aparece como sistema cerrado. El patriarcado, orden social real, se opone a su antítesis, el matriarcado, pero es un matriarcado puramente mítico, aunque es sentido como real por todos los hombres. Las mujeres son supuestamente ignorantes de su "existencia". A la vez, ellas son llevadas a defender un orden social que las coloca en un rango subordinado. Ideológicamente están controladas con los símbolos más temibles de la sociedad: los monstruos femeninos devoradores de hombres (Xalpen y Luna), a los que ellas también se oponen, pues una sociedad sin hombres es inconcebible (Chapman, 1986: 119).

\footnotetext{
${ }^{2}$ La autora aclara que los selk'nam no titulan sus mitos. Junto a las nominaciones que propone, incluye entre paréntesis las señaladas por Martín de Gusinde (El origen del kloketen de las mujeres y el origen del kloketen de los hombres) (Chapman, 1986: 104-112).
} 
El mito como construcción ideológica fue la forma más eficiente de mantener una lógica que determinó el papel social de los hombres y las mujeres selk'nam. Particularmente, la división de los poderes y el justificar el porqué lo mantienen. Chapman sostuvo que el hain no fue únicamente un rito religioso, también puede dar otras respuestas sociales, observables según la perspectiva que se seleccione para su explicación (sociológica, económica, psicológica, etc.). El grado de complejidad de la ceremonia va más allá, entonces, de un simple inventario de espíritus: la mujer indígena concentraba la ley del cosmos y, si la mujer no lograba recordar su pasado mítico, el orden de la sociedad patriarcal mantenía su lugar de poder.

Respecto a la elaboración textual de Chapman, son verificables sus diferenciaciones en comparación con las concesiones del género etnográfico. Por ejemplo, el movimiento del observador externo que incrementa la objetividad y la autoridad del investigador no fue una mecánica textual utilizada por la autora, ya que reconoce su cercanía con Lola y Ángela, sin encasillarlas de informantes, mujeres que compartieron el saber de su pueblo entre los intersticios cotidianos de su propia historia. En los constantes diálogos entre ellas se reafirmó un espacio cercano, asequible a través de otras posibilidades escriturales en el momento de trabajar con el registro de la memoria.

La representación etnográfica es una composición de carácter ficcional porque no describe una realidad independiente del etnógrafo, más bien desencadena la experiencia del autor frente a sus lectores. La escritura de Chapman no se diluyó entre las argumentaciones teóricas, sino acudió al recurso de la variación de narradores para autoafirmarse, porque su base fue la confianza que los selk'nam depositaron en ella. El circuito de lectura demostró la "verdad" de su representación, en la medida de que el otro no era presentado como objeto de estudio; pues, eran sujetos que comunicaban, por un lado, sus enseñanzas y, por otro, expresaban sus inquietudes existenciales ante la derrota más próxima (la muerte). Por lo mismo, la validación de la investigación de Chapman no recae en la solidez teórica (que existe), ni de ser mediadora o receptáculo del testimonio indígena, la autora se incluyó en cada escena, donde la familiaridad fue una característica muy presente a lo largo del texto:

¿A dónde fueron las mujeres que cantaban como los tamtam (canarios)? Había muchas mujeres. ¿A dónde se fueron?, me preguntó un día Lola Kiepja (Chapman, 1972: 145-158). 
El de curar se llamaba xo'on unitern, y Lola, como la gran mayoría de los chamanes, hombres y mujeres, lo poseía. Garibaldi a menudo fue paciente de Lola y también lo fui yo durante el último año de su vida (Chapman, 1972: 75).

Una estrategia común en el texto etnográfico es la de suprimir el sujeto informante para convertirlo en un espectador de las reflexiones teóricas del investigador, prueba fehaciente del grado de objetividad del discurso científico. No llegar a establecer contornos precisos entre hombres y mujeres o tipificar en atributos carente de un nombre son mecanismos que cooperaban en permitir la desaparición del individuo y en su reemplazo emerge el nativo objetivo: "crear una imagen de los individuos como entes abstractos que obedecían a impulsos colectivos y patrones de conductas mecánicas" (Poblete, 1999: 231). La antropóloga en cambio, en algunos pasajes del texto, colocó sus fuentes de estudios en el mismo nivel textual que Gusinde. Incluso en algunos puntos fueron citados a modos de una confrontación respecto a un tema específico:

[...] según Gusinde, el k'mal era lo que más se aproximaba a un jefe y había uno en cada familia extendida. Sostiene que el k'mal gozaba de un gran respeto por su edad madura, que sus consejos estaban respaldados por la experiencia y la profundidad de su juicio basado en el amplio conocimiento de la tradición, y que por su gran prestigio oral él podía mediar entre grupos antagónicos. Cuando existía la amenazada de una guerra, buscaban su consejo. Pero Ángela, Federico y Garibaldi insistieron en que, si bien se le reconocía como un gran guerrero, al que se honraba o temía, no era un líder moral ni tampoco un anciano respetado (Chapman, 1972: 77).

La cita anterior no apeló a desnaturalizar el trabajo de Gusinde en la Patagonia, el camino era discutir las instancias de mayor intervención del autor. La autora fue testigo de la interpelación que los mismos indígenas selk'nam expresaban cuando diferían de ciertas categorías descriptivas que los apropiaba como campo de estudio. Precisamente en una de las escenas textuales, Lola y Chapman revelan sus diferencias con la visión patriarcal. Dentro de las actividades diarias del hogar (espacio privado), Lola aludió a un acontecimiento público: la representación simbólica de la Luna. Seguidamente, Chapman aclaró las palabras de Lola en una transmisión de conocimiento entre mujeres, bajo una situación textual diferenciada; enseguida tomó de ejemplo una circunstancia similar entre Gusinde y los hombres, quienes explicaban el porqué de la aparición del eclipse. Así, se puede 
observar que el conflicto de género se puede sintetizar en esta imagen, en que las miradas dialogan en espacios y lenguajes particulares:

Una tarde de otoño, cuando estaba con Lola, ella me señaló con el dedo la enorme luna roja ardiendo sobre el horizonte, diciendo: "Kreeh háaten" (Luna está furiosa). Esta expresión significa también que la Luna ha entrado en eclipse. Los hombres contaron a Gusinde que Luna aparenta estar saciada cuando está llena, pero que es una artimaña para calmar los temores que provoca y poder atrapar desprevenidas a las personas, especialmente a los niños y comérselos (Chapman, 1972: 112).

La investigación de Chapman enunciaba la inquietud de estudiar al otro, con el objeto de responder críticamente a la construcción etnográfica tradicional que afrontó el lugar de la mujer indígena dentro de una posición falocéntrica. A pesar de que generó espacios significativos en la traducción de la memoria cultural de los últimos selk'nam, tales narraciones fueron trasplantadas hacia otra lectura exógena: la cuestión del género. La nominación de los mitos del matriarcado (mítico) y del patriarcado (plano real) explicitó términos que no pertenecieron ni al lenguaje ni a la concepción del mundo de las comunidades selk'nam. Entonces, fue un criterio que la autora aplicó en la búsqueda de herramientas conceptuales que lograran comprender las prácticas culturales como la ceremonia hain. Además, existieron circunstancias en que no pudo propiciar una explicación completa y profunda debido a la falta de datos; sin embargo, esos fragmentos de información no se constituyeron en un obstáculo para la autora, ya que analizó las huellas culturales de los indígenas a través de una expresión general de su organización (la extrapolación). En tal sentido, coincide con las concesiones del género etnográfico en lo que respecta a edificar un conocimiento total, subdivididos en unidades.

Por último, las transcripciones de los relatos de los selk'nam rematan en ciertos momentos en el texto, en un puente cortado. Las entrevistas a los indígenas mantuvieron siempre como eje los objetivos de la autora en tal sentido, la interrogación interrumpe una identidad contenida a pesar de la derrota, pero también visibiliza la incomprensión del observador frente a la resistencia del sujeto a contarlo todo, ya que sus muertos dialogan sólo con ellos, por ende es un espacio restringido a las contemplaciones ajenas. Por tanto, las aspiraciones de Chapman de concretizar un panorama cabal de los selk'nam fue interceptada por los mismos relatos (silenciados) que le habían sido negados:

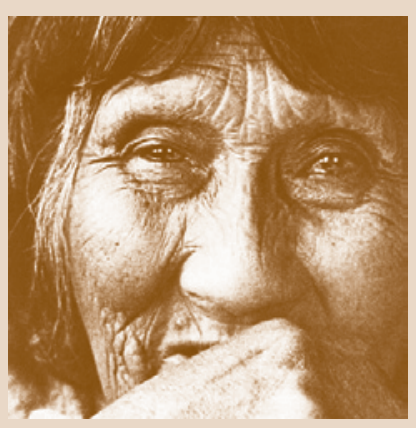

L. Kiepja 
Cierto día, cuando Ángela y Federico me comentaban cosas del hain, Ángela dejó la habitación por un momento y entonces Federico me dijo: "Aunque todo se terminó, Ángela es una mujer selk'nam y no debo hablar así enfrente de ella”. Y esto lo dijo cuarenta años después del último hain (Chapman, 1972: 118).

\section{REFERENCIAS}

Bridges, L. E. (1952). El último confín de la tierra. Traducción de Elena Cruz de Schwelm. Buenos Aires: Emecé.

Chapman, A. (1972). “Ensayo sobre algunos mitos y ritos selk'nam”. Museo del fin del mundo. Ushuaia: Argentina. (Título original: Lune en Terre de Feu. Mythes et rites des selk'nam. Tomo XII: 145-158).

Chapman, A. (1986). Los selk'nam. La vida de los onas. Buenos Aires: Emecé editores.

Gusinde, M. (1982). Los indios de Tierra del Fuego: resultado de mis cuatros expediciones en los años 1918 hasta 1924. Organizado bajo el auspicio del Ministerio de Instrucción Pública de Chile. Traducido de la edición austriaca por Werner Hoffmann. Revisión técnica por Olaf Blixen. Buenos Aires: Centro Argentino de Etnología Americana.

Marcus, G. y Fischer, M. J. (1986). Anthropology as Cultural Critique: An Experimental Moment in the Human Sciences. Chicago: University of Chicago Press.

Poblete, S. (1999). "La descripción etnográfica. De la representación a la ficción”. Cinta de Moebio 6: 212-248. 